\title{
A COMPACT EXTREME SCATTERING EVENT CLOUD TOWARD AO 0235+164
}

\author{
C. E. Senkbeil, S. P. Ellingsen, and J. E. J. Lovell \\ School of Mathematics and Physics, Private Bag 37, University of Tasmania, Hobart, TAS 7001, Australia \\ J.-P. MACQUART ${ }^{1}$ \\ NRAO, P.O. Box O, Socorro, NM 87801; and Department of Astronomy, California Institute of Technology, Pasadena, CA 91125 \\ G. Cimò \\ Joint Institute for VLBI in Europe, Postbus 2, 7990 AA Dwingeloo, Netherlands \\ AND \\ D. L. JAUNCEY \\ CSIRO Australia Telescope National Facility, Epping New South Wales, Australia \\ Received 2007 October 29; accepted 2007 November 27; published 2007 December 14
}

\begin{abstract}
We present observations of a rare, rapid, high-amplitude extreme scattering event toward the compact BL Lac object AO $0235+164$ at $6.65 \mathrm{GHz}$. The ESE cloud is compact; we estimate its diameter between 0.09 and $0.9 \mathrm{AU}$, with a distance of less than $3.6 \mathrm{kpc}$. Limits on the angular extent of the ESE cloud imply a minimum cloud electron density of $\sim 4 \times 10^{3} \mathrm{~cm}^{-3}$. Based on the amplitude and timescale of the ESE observed here, we suggest that at least one of the transients reported by Bower et al. may be attributed to ESEs.
\end{abstract}

Subject headings: BL Lacertae objects: individual (AO 0235+164) — galaxies: active - ISM: structure

Online material: color figures

\section{INTRODUCTION}

Since its discovery, the $z=0.940$ (Cohen et al. 1987) flatspectrum BL Lac object AO $0235+164$ has been known to exhibit variability (MacLeod et al. 1976) over a broad range of wavelengths and timescales (Raiteri et al. 2006 and references therein). Two absorbing galaxy complexes exist along the line of sight to this source at $z=0.524$ and $z=0.851$, respectively (Burbidge et al. 1976; Rieke et al. 1976), hindering interpretation of the spectrum and possibly contributing to the observed variability (e.g., by microlensing by stars in the foreground system; Ostriker \& Vietri 1985). The source is one of the most compact radio active galactice nuclei (AGNs); it exhibits long-term variability down to meter wavelengths and is slightly resolved by high angular resolution VLBI at $43 \mathrm{GHz}$ (Frey et al. 2000; Piner et al. 2006).

The extreme compactness of this source renders the interpretation of its variability difficult. For instance, there is debate on whether the origin of the centimeter-wavelength intraday variability in the observed $\mathrm{AO} 0235+164$ is primarily intrinsic or due to interstellar scintillation (ISS; Kraus et al. 1999; Lovell et al. 2003) and on what timescales each contributes. In addition, the source compactness renders it highly susceptible to flux density deviations by the compact intervening refracting structures in the local interstellar medium (ISM) that give rise to extreme scattering events (ESEs; Fiedler et al. 1987), because it is easy for even a small cloud to subtend the angular extent of such a small source.

Of the three main causes of centimeter-wavelength variability, ESEs are the most rare and certainly the least understood. Almost all flat-spectrum AGNs show variability (Altschuler \& Wardle 1977). The microarcsecond scintillation-induced variability (MASIV) survey found that $\sim 20 \%$ of this population exhibits scintillation at any one time, with over 56\% exhibiting ISS at any stage over the course of a year (Lovell et al. 2007; Jauncey

\footnotetext{
${ }^{1}$ NRAO Jansky Fellow.
}

et al. 2007). However, the estimated rate of ESEs in compact AGNs is only 0.013 source $^{-1} \mathrm{yr}^{-1}$ (Fiedler et al. 1987).

There are various plausible interpretations as to the origins of the structures that give rise to ESEs. These include intrinsically turbulent ionized clouds, purely refractive (Gaussian) lenses, and primarily neutral clouds enveloped by a thin ionized sheath that is responsible for the cloud's refractive properties (Fiedler et al. 1994; Romani et al. 1987; Clegg et al. 1998; Walker \& Wardle 1998). These models present a number of challenges; the electron densities implied by the plasma lens models require that the clouds be $\sim 10^{3}$ times overpressured with respect to the ambient ISM, whereas the neutral-cloud interpretation implies that such structures would contain a large fraction of the baryonic dark matter content of the Milky Way.

In this Letter, we report an unusually short-timescale ESE in AO $0235+164$. Our observations are reported in $\S 2$. In $\S 3$ we show that both intrinsic variability and interstellar scintillation are incapable of explaining this event, and we discuss the physical properties of the ESE necessary to give rise to the observed light curve. Our conclusions are presented in $\S 4$.

\section{OBSERVATIONS}

AO $0235+164$ has been monitored quasi-continuously since 2003 as part of the Continuous Single-Dish Monitoring of Intraday Variability at Ceduna (COSMIC) program (McCulloch et al. 2005). The observations were conducted by the use of the University of Tasmania Ceduna $30 \mathrm{~m}$ antenna at a center frequency of $6.65 \mathrm{GHz}$ with a $300 \mathrm{MHz}$ bandwidth. The flux density was sampled by scanning across the source in forward and reverse directions in both right ascension and declination. The flux density measurements are obtained from the height of the Gaussian profile of the source signal above the system temperature baseline. All the scans are scaled to a noise diode, which in turn is calibrated against $3 \mathrm{C} 227$, which, at this frequency, has a flux density of $1.99 \mathrm{Jy}$ (Baars et al. 1977). Measured pointing offsets are used to correct the amplitude in orthogonal scans, to minimize the impact of inaccurate pointing 


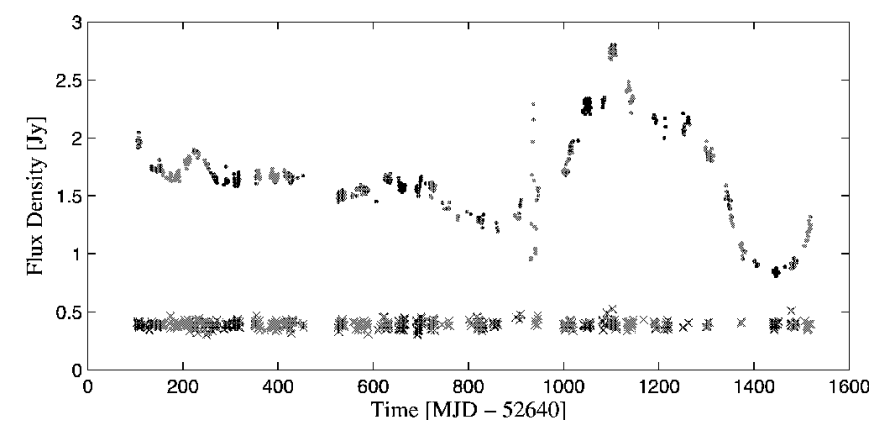

FIG. 1.-The $6.65 \mathrm{GHz}$ light curve of AO $0235+164$ (points) since 2003 January 1 (MJD 52,640). The corresponding light curve of the calibrator, 3C 277 , is shown (crosses), with $1.6 \mathrm{Jy}$ subtracted from its flux densities for display purposes. The averaging time for each flux density sample is $12 \mathrm{hr}$. [See the electronic edition of the Journal for a color version of this figure.]

on the measured amplitude. A correction is made to account for gain dependence of the antenna due to the distortion with respect to elevation. The four fitted amplitudes are averaged together to constitute a single calibrated sample.

The flux density monitoring data from Ceduna are known to suffer variations that are systematic in nature and predominantly diurnal in timescale, being related to changes in air temperature at the observatory. These errors scale with source flux density and affect source and calibrator equally (as they are approximately the same brightness). The amplitude of this systematic effect for the present case is $3 \%$, a factor $\sim 40$ times smaller than the variability in AO $0235+164$ discussed here. The calibrator data shown in Figure 1 demonstrate that this effect is negligible. Moreover, the variations occur on a much longer timescale than that of the diurnal systematic errors.

Figure 1 demonstrates that the source exhibited variations on timescales of days and months to years during the period 2003-2005. During the 5 day interval beginning 2005 July 20, the source exhibited a rapid, short-timescale increase in flux density from 1 to $2.3 \mathrm{Jy}$, followed by a fall to $1 \mathrm{Jy}$ over the next 5 days. Over the following 4 days, the flux density recovered to a value of $1.6 \mathrm{Jy}$, close to the long-term mean flux density of the source (Fig. 2).

This event is atypical of the variability commonly observed in this source and, indeed, in all seven of the variable flatspectrum quasars regularly observed in the COSMIC program. The peak amplitude of the flux density excursion exceeds the underlying mean by $44 \%$ and occurs on the exceedingly short timescale of only $\sim 4$ days. This behavior contrasts markedly with the long-term intrinsic variability evident in this source, as shown in Figure 1. This event is a one-off in the COSMIC data set; no similar events have been recorded in any of the other sources monitored in this program.

\section{ANALYSIS}

\subsection{Origin of the Event}

The main clue as to the physical origin of this event lies in the fact that it contains an excursion well below the average source flux density. Of the possible causes of this eventscintillation of a compact component, an intrinsic flare, microlensing by stars in an intervening galaxy, or an ESE in our Galaxy-only an ESE is capable of affecting the entire source brightness in such a way as to cause both the large positive and negative fluctuations observed.

In the month prior to the event, the light curve was flat, indicating that the flux density was dominated by quiescent emission originating from a region of the source that, we argue here, is too large to exhibit intrinsic variations on day timescales. If variations on a timescale of $\sim 40$ days are to be attributed to intrinsic variability, causality requires that the emission region encompass a size no larger than $\sim 0.03 \mathcal{D}(1+z)^{-1} \mathrm{pc}$, where $\mathcal{D}$ is the Doppler factor associated with the motion of the emission region toward the observer. However, the absence of interstellar scintillation in this source places a lower limit on the source size that is incompatible with this limit. Specifically, the absence of ISS implies an apparent angular size of $\gtrsim 20 \mu$ as. Using cosmological parameters of $H_{0}=71 \mathrm{~km} \mathrm{~s}^{-1} \mathrm{Mpc}^{-1}, \Omega_{M}=0.27$, and $\Omega_{\mathrm{vac}}=0.73$, the linear size of the emitting region must therefore exceed $0.16 \mathcal{D} \mathrm{pc}$. This size is not sufficiently small to allow causally connected intrinsic variations in the source on the timescale observed.

An interstellar scintillation-based explanation is unsatisfactory for the same reason. The onset of scintillation would require the appearance of a new source component compact enough to exhibit ISS. However, since ISS can only affect the flux density of this new compact component and not the extended quiescent emission, the light curve can never dip below the level of the quiescent emission.

Scattering due to the stellar wind of a nearby star along the line of sight to the source is also not a plausible explanation. We searched several Wolf-Rayet $\mathrm{O}$ and B star catalogs by use of the VizieR Online Data Catalog and found no nearby stars capable of generating bubbles of dense ionized matter with the properties required for this event.

Microlensing by stars in the intervening galaxies at $z=$ 0.524 and $z=0.851$ is similarly unable to explain the magnitude of the flux density excursion. The $50 \mu$ as source size

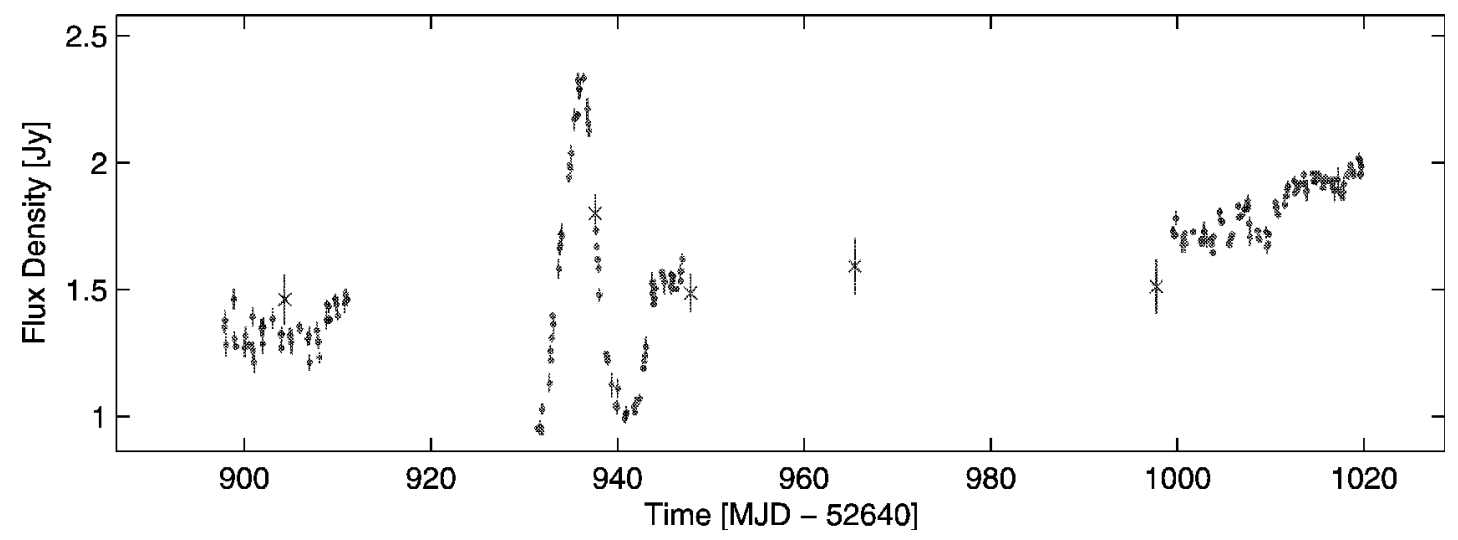

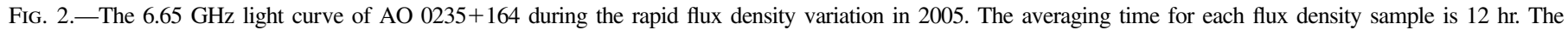
points marked by crosses denote $8 \mathrm{GHz}$ measurements reported by Bach et al. (2007). [See the electronic edition of the Journal for a color version of this figure.] 
makes it impossible for microlensing to appreciably alter the entire source flux density, because lensing only affects a region of size comparable to the Einstein radius. Even for the closer of the two systems, the Einstein radius of a star of mass $m$ is only $\approx 1.56\left(\mathrm{~m} / \mathrm{M}_{\odot}\right)^{1 / 2} \mu$ as (assuming $H_{0}=70 \mathrm{~km} \mathrm{~s}^{-1} \mathrm{Mpc}^{-1}$ ). Futhermore, we note that the observed dip in the source flux density during the event is at variance with the magnification profile expected from a microlensing event. A more detailed argument against the likelihood of microlensing in $\mathrm{AO}$ $0235+164$ is presented in Kraus et al. (1999).

We are thus forced to conclude that only an event completely extrinsic to the source is capable of affecting the source's emission, which is too large to exhibit ISS or to be causally connected to an intrinsic flare. Therefore, the most plausible explanation is that the flux density excursion is caused by an ESE. We also note that the light curve exhibits a large increase and a subsequent sharp decrease that are characteristic of ESEs. This is consistent with the passing of the cloud across the line of sight, in which the radiation is first focused on the observer and then, as the observer passes the ESE boundary, is steered out of the line of sight into the neighboring focusing region.

\subsection{ESE Cloud Properties}

The duration of the ESE places constraints on the transverse length scale of the ionizing material. The absence of data prior to and following the high-amplitude variations places uncertainty on the total length of the ESE. We argue that the event had ceased by day 947 . This is supported by the $8 \mathrm{GHz}$ data from Medicina (Bach et al. 2007) that agree well with the Ceduna data, when they overlap, and, following the period in which rapid changes were observed, these data are consistent with the flux density having returned to the underlying slow intrinsic change. The earliest possible date for the commencement of the event is day 916. Thus, the maximum likely event duration is $\Delta T=$ [947-916] days, whereas the minimum possible duration is 16 days, assuming that the event is temporally symmetric about its peak, as is observed in other ESEs (e.g., Fiedler et al. 1987).

The transverse extent of the cloud is

$$
r=0.18\left(\frac{v}{20 \mathrm{~km} \mathrm{~s}^{-1}}\right)\left(\frac{\Delta T}{16 \text { days }}\right) \mathrm{AU},
$$

where $v$ is the speed of the cloud transverse to the line of sight, plausibly in the range $10-50 \mathrm{~km} \mathrm{~s}^{-1}$. Taking the uncertainties in both $\Delta T$ and $v$ into account, the estimated linear scale of the cloud is in the range 0.09-0.90 AU.

Since the event significantly alters the entire flux density of the source, the angular extent of the ionized cloud must be comparable to or greater than the angle subtended by the core of the source. Space VLBI observations of AO $0235+156$ place an upper limit on the core size at $4.8 \mathrm{GHz}$ of $50 \mu$ as (Frey et al. 2000). This core size, combined with the favored cloud size of $0.18 \mathrm{AU}$, requires a cloud distance of less than $3.6 \mathrm{kpc}$.

The electron density of an ESE cloud must be much higher than the ambient medium. A simple estimate of the electron density is obtained by considering refraction by a cloud with a Gaussian density profile:

$$
n_{e}=n_{0} \exp \left[\frac{-\left(r^{2}+z^{2}\right)}{2 R_{0}^{2}}\right]
$$

where $n_{0}$ is the maximum electron density, $R_{0}$ is the cloud radius, $z$ is the distance along the line of sight, and $r$ is the distance from the cloud center transverse to the line of sight. The bending angle, $\alpha$, of the cloud must be comparable to the angular size of the source, so that

$$
\Delta \theta \approx \alpha=\frac{1}{k} \frac{\partial \phi}{\partial r}
$$

where $k$ is the wavenumber and $\phi$ is the phase delay imparted by the cloud. These assumptions lead to a relationship between $n_{0}$ and $\alpha$ as a function of distance, $r$, from the cloud center:

$$
\alpha=(2 \pi)^{1 / 2} n_{0} r_{e} \lambda \frac{r}{R_{0}} \exp \left(-\frac{r^{2}}{2 R_{0}^{2}}\right),
$$

where $r_{e}$ is the classical electron radius. The maximum bending angle occurs at $r=R_{0}$; this requires a minimum cloud electron density of $4.1 \times 10^{3} \mathrm{~cm}^{-3}$, comparable to or higher than density estimates of previous ESE clouds (Fiedler et al. 1987).

The COSMIC program, to date, observed seven sources over a period of 4 years, during which time one ESE was detected. Our observation of a single event in 35 source years of monitoring is not inconsistent with the ESE rate inferred by Fiedler et al. (1987). This supports our interpretation of this phenomenon as an ESE.

There have been a number of recent reports of short-duration transients at high Galactic latitude (Bower et al. 2007; Niinuma et al. 2007; Matsumura et al. 2007). It is interesting to speculate whether short-duration ESEs similar to, or perhaps even more extreme than, the event we have observed in AO $0235+164$ could be responsible for these events. Bower et al. (2007) used archival VLA calibration observations to investigate the presence of radio transients in a high galactic latitude field for a period of about 20 minutes, on average, once every 7 days for more than 20 years. They identified 10 radio transient events in which there is no persistent emission stronger than a few microjanskys. However, in addition to these events, their data show four cases in which a source detected in the deep radio images was detected in only a single-epoch 20 minute observation. Comparing the peak and mean flux densities, the amplification observed in these events is of the order of 2-5, larger than typical ESEs, but not implausibly so. These events may then be due to ESEs with timescales that are similar to the AO $0235+168$ event ( $<20$ days). However, for the transients of Bower et al. (2007) and for those detected in the Nasu $1.4 \mathrm{GHz}$ observations (Niinuma et al. 2007; Matsumura et al. 2007), which have no detected persistent radio emission, the implied amplifications are of the order of 10-100 or more and cannot plausibly be explained by ESEs.

\section{CONCLUSION}

We observed a rapid 16 day timescale flux density variation in $\mathrm{AO} 0235+164$ as part of the $6.7 \mathrm{GHz}$ COSMIC program. The presence of a flux density excursion below the underlying mean flux density of the source is consistent with an ESE cloud passing through the line of sight.

Assuming an ESE cloud velocity of $20 \mathrm{~km} \mathrm{~s}^{-1}$, we estimate that the cloud has a linear scale size ranging from 0.09 to $0.90 \mathrm{AU}$, with a distance of less than $3.6 \mathrm{kpc}$. We estimate that the minimum electron density needed for the cloud to produce an ESE is $4 \times 10^{3} \mathrm{~cm}^{-3}$.

The detection and characterization of this ESE required the intensive flux density monitoring by the COSMIC program. The rapidity of this ESE suggests that perhaps other rapid ESEs 
may be overlooked in other monitoring programs, due to undersampling of the events.

This research has been supported by ARC grant DP0342500 at the University of Tasmania. We thank Bev Bedson for her vital contribution to the operation of the Ceduna observatory and the COSMIC project. We thank Stefan Dieters for searching the archives of satellite-based observatories for events coincident with the ESE detection. The National Radio Astronomy Observatory is a Facility of the National Science Foundation operated under cooperative agreement by Associated Universities, Inc. This research was supported in part by NASA grant JPL 1253153 to the University of Minnesota.

\section{REFERENCES}

Altschuler, D. R., \& Wardle, J. F. C. 1977, MNRAS, 179, 153

Baars, J. W. M., Genzel, R., Pauliny-Toth, I. I. K., \& Witzel, A. 1977, A\&A, 61,99

Bach, U., et al. 2007, A\&A, 464, 175

Burbidge, E. M., Caldwell, R. D., Smith, H. E., Liebert, J., \& Spinrad, H. 1976, ApJ, 205, L117

Bower, G. C., Saul, D., Bloom, J. S., Bolatto, A., Filippenko, A. V., Foley, R. J., \& Perley, D. 2007, ApJ, 666, 346

Clegg, A.W., Fey, A. L., \& Lazio, T. J. W. 1998, ApJ, 496, 253

Cohen, R. D., Smith, H. E., Junkkarinen, V. T., \& Burbidge, E. M. 1987, ApJ, 318, 577

Fiedler, R., Dennison, B., Johnston, K. J., Waltman, E. B., \& Simon, R. S. 1994, ApJ, 430, 581

Fiedler, R. L., et al. 1987, ApJS, 65, 319

Frey, S., et al. 2000, PASJ, 52, 975

Jauncey, D. L., et al. 2007, Astron. Astrophys. Trans., in press

Kraus, A., et al. 1999, A\&A, 344, 807
Lovell, J. E. J., Jauncey, D. L., Bignall, H. E., Kedziora-Chudczer, L., Macquart, J.-P., Rickett, B. J., \& Tzioumis, A. K. 2003, AJ, 126, 1699

Lovell, J. E. J., et al. 2007, ASP Conf. Ser. 365, SINS-Small Ionized and Neutral Structures in the Diffuse Interstellar Medium, ed. M. Haverkorn \& W. M. Goss (San Francisco: ASP), 279

MacLeod, J. M., Andrew, B. H., \& Harvey, G. A. 1976, Nature, 260, 751

Matsumura, N., et al. 2007, AJ, 133, 1441

McCulloch, P., Ellingsen, S.P., Jauncey, D.L., Carter, S. J. B., Cimò, G., Lovell, J. E. J., \& Dodson, R. G. 2005, AJ, 129, 2034

Niinuma, K., et al. 2007, ApJ, 657, L37

Ostriker, J. P., \& Vietri, M. 1985, Nature, 318, 446

Piner, B. G., Bhattarai, D., Edwards, P. G., \& Jones, D. L. 2006, ApJ, 640, 196

Raiteri, C. M., et al. 2006, A\&A, 459, 731

Rieke, G. H., Grasdalen, G. L., Kinman, T. D., Hintzen, P., Wills, B. J., \& Wills, D. 1976, Nature, 260, 754

Romani, R.W., Blandford, R. D., \& Cordes, J. M. 1987, Nature, 328, 324

Walker, M. A., \& Wardle, M. 1998, ApJ, 498, L125 